$>$ La leptine, produit du gène $o b$, est une protéine de 16 kDa qui agit par des mécanismes centraux pour contrôler l'équilibre énergétique. Outre le tissu adipeux, d'autres organes dont l'estomac produisent également la leptine. Les récepteurs de la leptine ont été identifiés dans le tractus digestif, notamment dans l'estomac, l'intestin, le foie, le pancréas. Les études récentes suggèrent que la leptine gastrique puisse agir localement sur de nouvelles cibles pour contrôler les fonctions intestinales d'absorption, de sécrétion et de développement. De plus, elle pourrait jouer un rôle dans la physiopathologie intestinale. Cet article résume les données expérimentales suggérant que la leptine soit une nouvelle hormone du tractus gastro-intestinal. <

\section{Effets paracrines de la leptine produite par l'estomac}

Marion Buyse, Thomas Aparicio, Sandra Guilmeau, Hélène Goïot, Iradj Sobhani, André Bado

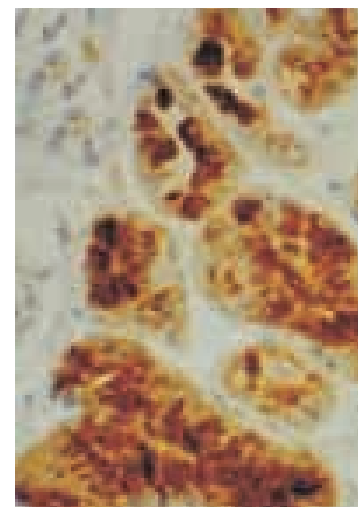

furent ensuite identi-
Inserm U.410, IFR02 Claude Bernard, UFR Xavier Bichat, 16, rue Henri Huchard, BP 416, 75870 Paris Cedex 18, France. bado@bichat.inserm.fr fiés comme des lieux de synthèse et de sécrétion de la leptine, suggérant de nouvelles cibles pour cette hormone. La mise en évi-

La leptine est une protéine de 16 kDa qui a été largement décrite pour son rôle majeur dans la régulation de l'équilibre énergétique [1]. Ainsi, chez les souris ob/ob déficientes en leptine biologiquement active, l'administration de leptine recombinante provoque une réduction de la prise alimentaire et une augmentation des dépenses énergétiques [2]. La résultante de ces effets est une réduction de leur obésité. Cette action «antiobésité» de la leptine met en jeu une modulation de l'expression et de la sécrétion de peptides orexigènes et anorexigènes via l'activation de récepteurs de la leptine au niveau de l'hypothalamus [3, 4]. En effet, le gène $d b$ codant pour le récepteur de la leptine a été identifié [4] un an après le gène $o b[1]$. Ce récepteur possède un seul domaine transmembranaire et appartient à la famille des récepteurs de cytokines de classe I. Sept isoformes résultant d'un épissage alternatif de l'ARN primaire $d b$ ont été décrites [5]. Elles se distinguent entre elles par la longueur de leur domaine intracellulaire.

Le tissu adipeux a été initialement considéré comme l'unique source de production de la leptine. Le placenta [6], les muscles squelettiques [7] et l'estomac [8] dence de la leptine gastrique et de ses récepteurs dans l'estomac, l'intestin, le pancréas, le foie, et le fait que le tractus gastro-intestinal soit la première interface rencontrée par les aliments après leur ingestion, suggèrent fortement que la leptine est une hormone du tractus gastro-intestinal.

\section{L'estomac synthétise et sécrète la leptine}

L'estomac joue un rôle essentiel dans le stockage et le broyage des aliments, préparant ainsi les nutriments à la digestion et à l'absorption intestinale. La muqueuse fundique de l'estomac est formée d'une monocouche de cellules présentant de nombreuses invaginations au fond desquelles s'ouvrent des glandes constituées par des cellules à mucus, des cellules pariétales, des cellules principales et des cellules endocrines (Figure 1). Cet épithélium fundique de l'estomac synthétise et sécrète la leptine chez le rat [8] et chez l'homme [9]. Les cellules produisant la leptine correspondent aux cellules principales sécrétant également le pepsinogène (Figure 1). La sécrétion de la leptine gastrique est contrôlée par des 
facteurs nerveux [10] et hormonaux activés par le repas $[8,9]$. Son expression est augmentée dans la gastrite à Helicobacter pylori et dans les ulcères expérimentaux, suggérant un rôle physiopathologique.

La leptine produite par l'estomac se distingue de la leptine adipocytaire par sa mobilisation rapide au cours du repas [11] et sa sécrétion principalement exocrine [8]. Ce pool gastrique de leptine, qui n'est pas totalement dégradé dans le suc gastrique (même à $\mathrm{pH} 2$ ), transite par le duodénum sous forme libre et liée à des macromolécules de haut poids moléculaire [12] et atteint le côlon sous forme biologiquement active. La différence dans la cinétique de sécrétion postprandiale implique des systèmes de régulation et des fonctions différentes pour ces deux pools de leptine. La sécrétion rapide, à partir du pool gastrique, servirait à la gestion efficace de l'absorption intestinale des nutriments et la sécrétion tardive, à partir du pool adipocytaire, à celle du stockage et de l'utilisation des réserves énergétiques.

La présence des récepteurs de la leptine le long de l'intestin et leur localisation à l'apex des entérocytes [13, 14] indiquent qu'ils sont des cibles privilégiées de la leptine circulant dans la lumière intestinale. Nous avons énoncé trois hypothèses d'action dont deux impliquent des mécanismes paracrines: (1) la leptine gastrique passe dans la circulation porte, est véhiculée jusqu'au foie où elle contrôle le métabolisme hépatique; (2) la leptine gastrique agit localement dans l'estomac en activant les afférences vagales viscéro-sensitives pour produire des signaux de satiété qui sont intégrés et traités dans le système nerveux central (SNC); (3) la leptine gastrique gagne l'intestin où elle interagit avec ses récepteurs pour contrôler les fonctions d'absorption, de sécrétion et de développement de l'épithélium intestinal. La suite de cet article détaillera particulièrement les principaux résultats en faveur des deux dernières hypothèses.

\section{La leptine gastrique: facteur de satiété via une action neurocrine locale}

Dès 1997, il avait été rapporté que la coadministration périphérique de leptine et de cholécystokinine (CCK), à des doses qui n'ont pas d'effet sur la prise alimentaire, induisait une satiété précoce chez la souris [15]. Cette action synergique était dépendante de l'intégrité des fibres viscéro-sensitives du nerf vague. L'identification de deux types de fibres vagales sensibles à la leptine, dont le type 2 nécessitant pour son activation une sensibilisation préalable à la CCK-8, renforçait l'idée selon laquelle le nerf vague était le site d'action de cette interaction. C'est ainsi que l'expression des récepteurs de la leptine a été mise en évidence dans le ganglion plexiforme contenant les corps cellulaires des neurones afférents viscéro-sensitifs du nerf vague chez le rat [16] et chez I'homme [17]. Ces récepteurs sont colocalisés avec le facteur de transcription STAT3 (signal transducer and activator of transcription 3), élément majeur de la signalisation intracellulaire de la leptine. II était alors tentant de proposer un modèle d'action (Figure 2) dans lequel la leptine gastrique libérée par le repas active localement ses récepteurs situés dans les terminaisons nerveuses pour induire des signaux de satiété transmis au SNC par les afférences vagales. Ce modèle est en accord avec les données montrant que l'administration de leptine dans l'estomac augmente l'activité électrique des neurones du faisceau du tractus solitaire

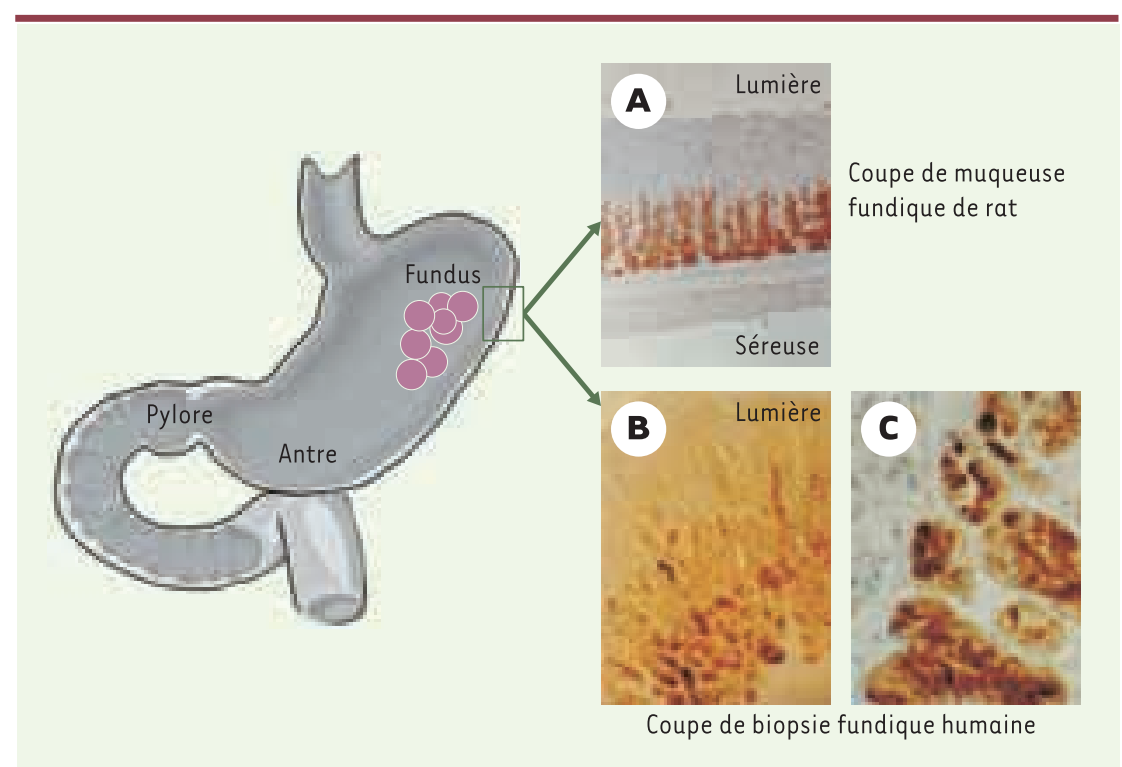

Figure 1. Anatomie de l'estomac et immunohistochimie de la leptine gastrique. L'immunoréactivité de la leptine est localisée dans la muqueuse fundique chez le rat et chez l'homme. A. Réaction immunohistochimique dans la muqueuse fundique de rat avec un anticorps polyclonal antileptine de souris (coupe fixée au paraformaldéhyde $4 \%$ ). L'immunoréactivité de la leptine est visible dans l'épithélium glandulaire, exclusivement dans le fond des glandes fundiques. Une telle localisation correspond essentiellement à la distribution des cellules principales qui synthétisent et sécrètent également le pepsinogène. $B$. Réaction immunohistochimique dans la muqueuse fundique humaine (faible grossissement) avec un anticorps polyclonal anti-leptine humaine (coupe fixée au paraformaldéhyde $4 \%$ ). L'immunoréactivité de la leptine est visible dans l'épithélium glandulaire, localisation similaire à celle observée chez le rat. $C$. Fort grossissement de $\boldsymbol{B}$ montrant des cellules principales fortement immunoréactives à la leptine. 
(NTS) (principal site bulbaire de projection des afférences vagales) et l'expression de la protéine c-Fos (marqueur d'activité neuronale) dans le noyau paraventriculaire de l'hypothalamus [18]. Cette action locale de la leptine gastrique amplifierait l'action des récepteurs de la cholécystokinine CCK-l précédemment décrits dans le nerf vague, expliquant ainsi l'effet synergique de la CCK et de la leptine sur l'activité neuronale du NTS et sur l'induction précoce de la satiété. Cette action synergique est renforcée par la capacité de la leptine d'augmenter les concentrations circulantes de CCK en acti-

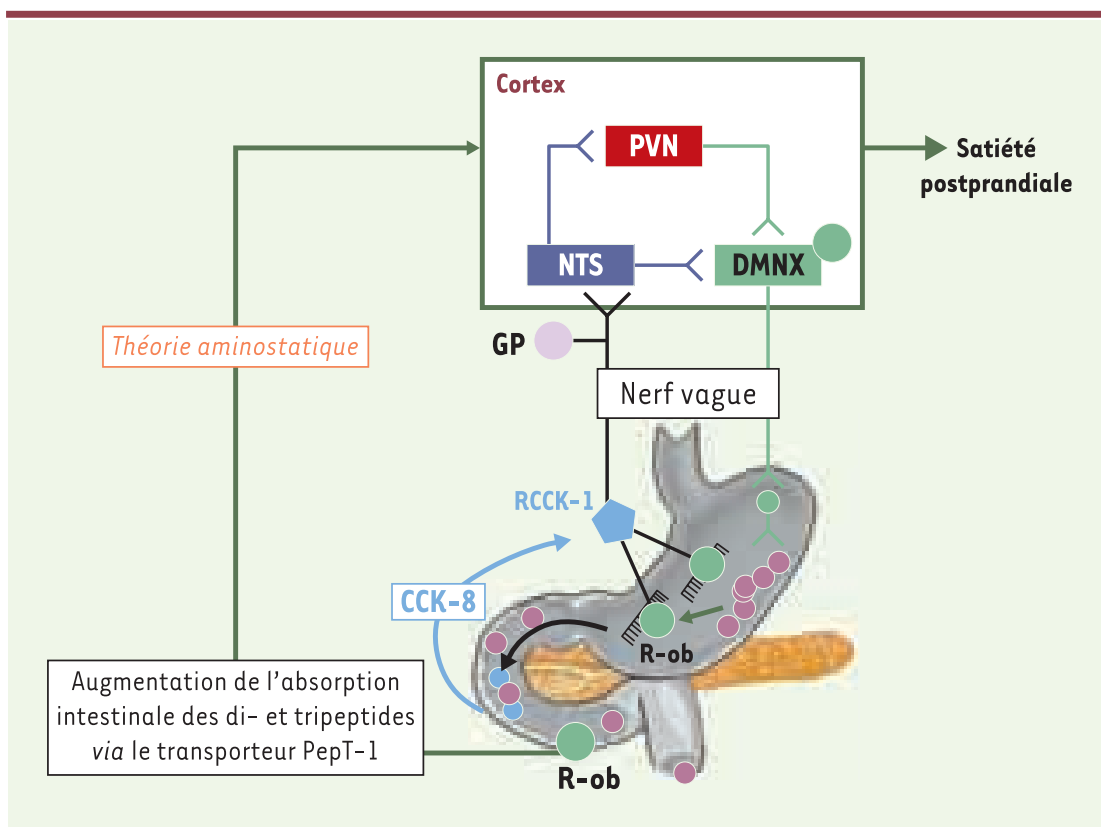

Figure 2. Modèle hypothétique d'action de la leptine gastrique. La leptine gastrique induit la satiété postprandiale par deux voies d'action: une action neurocrine via l'activation de ses récepteurs localisés dans les afférences viscéro-sensitives vagales et une action paracrine via une augmentation de l'absorption intestinale des protéines par l'intermédiaire du transporteur intestinal PepT-1. La leptine gastrique libérée par le repas (petits cercles roses) active localement ses récepteurs R-ob (cercles verts) situés dans les terminaisons nerveuses pour produire des signaux de satiété transmis au système nerveux central par les afférences vagales. Les signaux déclenchés par l'activation des fibres vagales sont intégrés dans le faisceau du tractus solitaire (NTS). Cette action locale de la leptine gastrique amplifierait l'action des récepteurs de la cholécystokinine (RCCK-1) expliquant ainsi l'effet synergique de la cholécystokinine (CCK, petits cercles bleus) et de la leptine sur l'activité neuronale du NTS et sur l'induction précoce de la satiété (la cholécystokinine est un facteur de satiété produit par les cellules endocrines intestinales). À partir du NTS, les signaux se projetteraient dans le noyau moteur dorsal du nerf vague (DMNX, siège des corps cellulaires des neurones moteurs) pour assurer un contrôle parasympathique des fonctions viscérales par un réflexe vago-vagal. Alternativement, ces signaux pourraient se projeter dans le noyau paraventriculaire de l'hypothalamus (PVN) pour contrôler la prise alimentaire. GP: ganglion plexiforme. CCK-8: extrémité carboxy-terminale biologiquement active de la cholécystokinine. La leptine présente dans la lumière jéjunale augmente l'absorption des di- et des tripeptides via le transporteur intestinal dépendant des protons, PepT1. Selon la théorie aminostatique [27], des changements dans la concentration plasmatique des acides aminés sont détectés par le cerveau et influencent la prise alimentaire. déficients en système leptinique fonctionnel. Ces protéines jouent un rôle essentiel dans l'exocytose des neurotransmetteurs qui se fait par la fusion de vésicules synaptiques à la membrane présynaptique et donc dans leur libération par les terminaisons présynaptiques. Bien que des travaux soient encore nécessaires, il est raisonnable de penser que ces altérations de l'activité nerveuse sont liées à une diminution et/ou à un blocage de la libération de neurotransmetteurs dans la fente synaptique, du fait de l'absence de système leptinique fonctionnel.

\section{La leptine digestive contrôle l'absorption des nutriments}

L'intestin est le site principal de l'absorption des nutriments. Dans l'intestin, le chyme subit l'action du suc pancréatique, de la bile, poursuivant les processus de digestion entamés par le suc gastrique. Les aliments ainsi dégradés en éléments plus petits par les enzymes pancréatiques et intestinales franchissent la barrière intestinale soit par un transport actif, soit par une diffusion passive, soit par une diffusion facilitée qui nécessite également des protéines de transfert. Quelques données impliquent la leptine circulante (reflet de la leptine adipocytaire) dans la réduction de l'absorption intestinale des lipides $[19,20]$ et des sucres [21]. À l'heure actuelle, la leptine digestive n'a été impliquée que dans le contrôle de l'absorption des protéines [12].

L'absorption des résidus protéiques est assurée par un transporteur dépendant 
des protons, PepT-1, qui transfère les di- et tripeptides de la zone de faible activité dipeptidasique (lumière intestinale) vers la zone de forte activité (cytoplasme des entérocytes). PepT-1, dont le gène a été cloné en 1994, est une protéine de 80 kDa possédant 12 domaines transmembranaires, dont une large boucle hydrophile extracellulaire entre les domaines transmembranaire 9 et 10 [22]. PepT-1 est exprimé dans le duodénum, le jéjunum et l'iléon. Il est absent de l'œsophage, de l'estomac, du côlon et du rectum. Dans le jéjunum, où il est le plus fortement exprimé, PepT-l est localisé dans la bordure en brosse avec un gradient d'expression croissant suivant l'axe crypto-villositaire.

Chez le rat in vivo, l'administration de leptine dans la lumière jéjunale augmente l'absorption des di- et des tripeptides via PepT-1 [13]. Ces résultats ont été étayés in vitro sur les cellules Caco2 qui se différencient spontanément en entérocytes et qui expriment les différentes formes du récepteur de la leptine. Cette action à court terme de la leptine résulte essentiellement de la mobilisation du pool intracellulaire de PepT-1 à la membrane plasmique (Figure 3). Dans les conditions physiologiques, cette action de la leptine gastrique constituerait un nouveau mécanisme par lequel la leptine contrôlerait les réserves énergétiques. Dans les conditions physiologiques, elle favoriserait notamment l'absorption intestinale des protéines au détriment de celle des lipides. Le dérèglement de ce mécanisme pourrait participer à la mise en échec du système de régulation des réserves énergétiques et conduire à l'obésité.

PepT-l est également impliqué dans l'absorption intestinale de médicaments à structure peptidomimétique (inhibiteur de l'enzyme de conversion, antibiotiques de la famille des $\beta$-lactames et certaines anti-protéases). L'augmentation de son activité par la leptine intestinale pourrait donner lieu à des développements touchant à l'amélioration de la biodisponibilité des médicaments.

\section{Rôle de la leptine dans les maladies inflammatoires de l'intestin}

Un des rôles émergents de la leptine est celui qu'elle pourrait jouer dans la régulation de l'inflammation intestinale [23]. En effet, les souris ob/ob déficientes en leptine fonctionnelle sont résistantes à l'induction d'une colite expérimentale. Cela est en parfait accord avec l'altération des réponses immunitaires et inflammatoires observée chez ces animaux. Bien que l'administration de la leptine convertit la résistance de ces souris en susceptibilité, le caractère pro- ou antiinflammatoire de la leptine reste toujours discuté.

Une des caractéristiques des maladies inflammatoires chroniques de l'intestin ( $\mathrm{MICl}$ ) est la perturbation de l'équilibre entre les cytokines pro- et anti-inflammatoires souvent associée à un important infiltrat inflammatoire et à une altération de la muqueuse colique. Or, chez des sujets atteints de rectocolite hémorragique, la leptine est détectée dans les lavages coliques à des concentrations supérieures à celles retrouvées chez les sujets sains [24]. Cette augmentation de la leptine colique est associée à l'apparition d'un immunomarquage leptin-like dans les colonocytes. Un tel résultat peut être rapproché de la capacité des cellules épithéliales à produire et à sécréter des cytokines participant ainsi directement au développement de l'inflammation intestinale. La présence des récepteurs de la leptine à

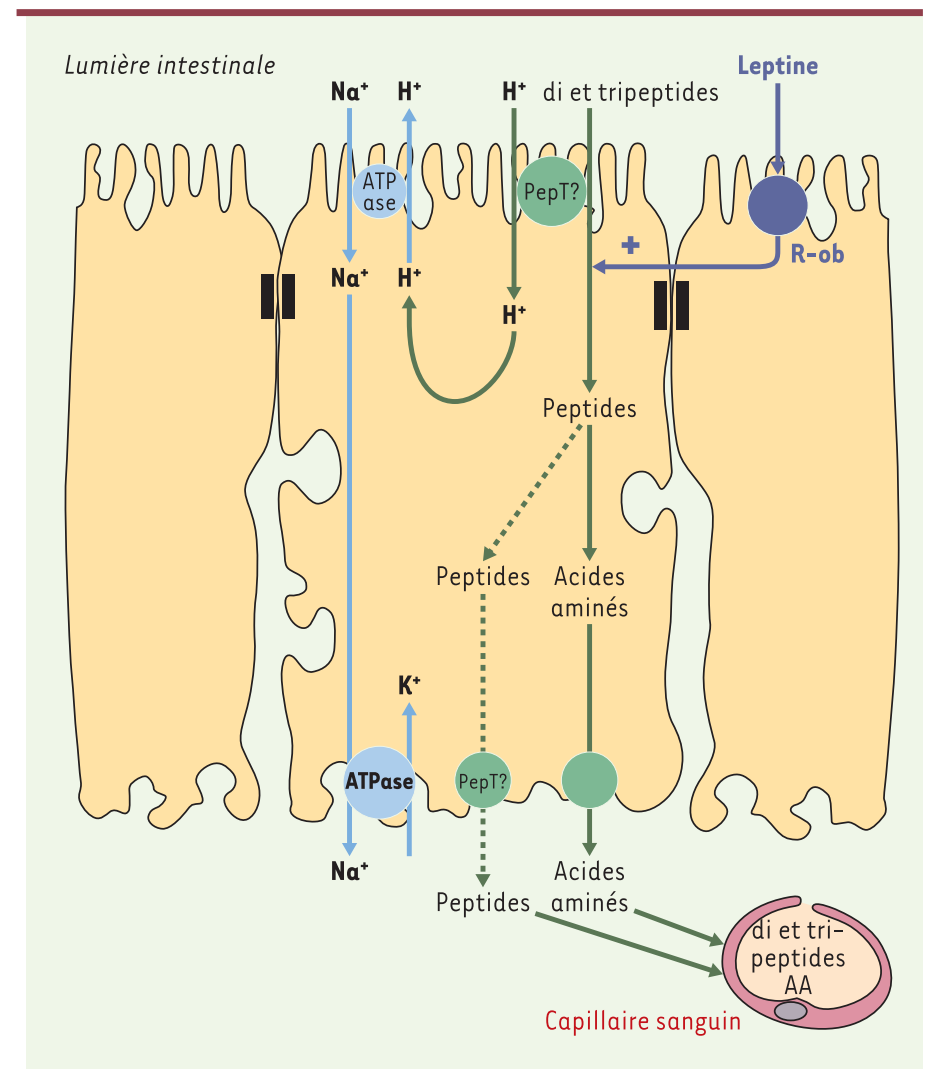

Figure 3. Mécanisme général du transport par le transporteur intestinal PepT-1 et son contrôle par la leptine. Les di- et les tripeptides de même que les médicaments peptidomimétiques sont absorbés au pôle apical de l'entérocyte par un cotransport avec un ion $\mathrm{H}^{+}$. Le gradient de protons est produit et maintenu par un antiport $\mathrm{Na}^{+} / \mathrm{H}^{+}$dans la membrane de la bordure en brosse, grâce à un gradient entrant de $\mathrm{Na}^{+}$. $\mathrm{La} \mathrm{Na}^{+}, \mathrm{K}^{+}$-ATPase de la membrane basolatérale permet la sortie active de $\mathrm{Na}^{+}$, ce qui réduit la concentration intracellulaire et permet l'établissement d'un gradient entrant de $\mathrm{Na}^{+}$. La leptine gastrique atteignant la lumière intestinale active les récepteurs de la leptine ( $R$-ob) situés à l'apex des entérocytes et stimule l'absorption intestinale des résidus protéiques via une mobilisation du pool cytoplasmique du transporteur PepT-1 à la membrane. AA: acides aminés. 
l'apex des colonocytes, et l'activation du facteur de transcription NF-KB par la leptine dans les cellules intestinales $\mathrm{Caco-2}$, suggèrent que la leptine soit impliquée dans la physiopathologie des $\mathrm{MICl}$. De plus, la leptine contrôle l'absorption colique du butyrate, source majeure d'énergie pour le colonocyte via la voie de la $\beta$-oxydation.

Dans la lumière du côlon, les sucres complexes contenus dans les fibres alimentaires sont dégradés par fermentation bactérienne en acides gras à chaîne courte, principalement en butyrate, en propionate et en acétate. Dans les $\mathrm{MICl}$, en particulier dans la rectocolite hémorragique, la consommation de butyrate est diminuée de moitié, sans doute en raison d'une altération de la capacité des colonocytes à absorber cet acide gras. Le transport actif du butyrate est assuré par le transporteur de monocarboxylates de type 1 (MCT-1). Il a été montré que la leptine, appliquée au pôle apical des cellules intestinales Caco-2, est capable d'augmenter l'absorption du butyrate via MCT-1 par des mécanismes mettant en jeu une augmentation du pool intracellulaire de MCT- 1 et la translocation du complexe MCT-1/CD147 à la membrane apicale des colonocytes [25]. Notons que l'interaction de MCT-1 avec CD147 (appartenant à la superfamille des immunoglobulines) est indispensable au ciblage de MCT-l à la membrane plasmique [26]. L'augmentation de l'absorption du butyrate par l'intermédiaire du complexe MCT-1/CD147 est une donnée importante, car le butyrate a été impliqué dans la prolifération, la différenciation et la mort cellulaires. Plus récemment, nous avons démontré que la leptine empêchait la mort cellulaire des cellules épithéliales intestinales induite par le butyrate. II est donc possible que l'altération du contrôle de l'expression du complexe MCT-1/CD147 par la leptine, dans les muqueuses inflammatoires, affecte les événements intracellulaires contrôlant la prolifération et l'apoptose des cellules épithéliales coliques. Une meilleure connaissance du rôle de la leptine colique dans les $\mathrm{MICl}$ devrait permettre d'élaborer des stratégies de thérapie pharmacologique utilisant la production locale de leptine ou le blocage de son action dans l'intestin par des probiotiques (bactérie lactique ou flore intestinale modifiée, Lactobacillus...) produisant des substances agonistes ou antagonistes de la leptine.

\section{Conclusions}

Le rôle physiologique de la leptine produite par l'estomac n'est pas encore totalement élucidé. Cependant, il apparaît de plus en plus clairement que son rôle ne se limite pas au déclenchement de la satiété postprandiale, mais concerne également le maintien de l'homéostasie de l'épithélium gastro-intestinal en contrôlant ses fonctions de réparation-protection, de développement, de sécrétion et d'absorption. À n'en pas douter, une meilleure connaissance du rôle physiologique de la leptine digestive, de son statut dans l'obésité et dans les maladies gastro-intestinales devraient permettre d'engager des stratégies de développement de molécules actives dans le traitement de ces maladies. $\diamond$

\section{SUMMARY}

Paracrine actions of the stomach-derived leptin

Leptin, a 16 kilodalton protein-encoded by the ob gene, is involved in the regulation of food intake, body composition, and energy expenditure through a central feedback mechanism. Initially thought to be adipocyte-specific, the ob gene, as well as the leptin receptor, has been found in a variety of other tissues. Relevant to this review, the leptin gene and its receptor have been identified in the stomach, intestine, liver, and pancreas. Recent data also suggest that gut leptin may act locally within the gastrointestinal tract to influence intestinal functions such as nutrient absorption and may have a physiopathological implication. This review emphasises the concept that leptin may be a new gastrointestinal hormone.

\section{REMERCIEMENTS}

Les travaux provenant de notre laboratoire ont été soutenus par l'Inserm, l'APEX99 $n^{\circ} 4 X 006 \varepsilon$ (A. Bado), Nestlé France SA, l'Association pour la Recherche sur le Cancer (ARC) et la Ligue Nationale contre le Cancer, subvention n० 75/01-EDT/06. Les auteurs expriment leurs remerciements à Jean-Pierre Laigneau pour son aide précieuse dans la réalisation des illustrations. $\diamond$ 


\section{RÉFÉRENCES}

1. Zhang $Y$, Proenca R, Maffei M, Barone M, Leopold L, Friedman JM. Positional cloning of the mouse obese gene and its human homologue. Nature 1994 ; 372: 425-32.

2. Halaas JL, Gajiwala KS, Maffei M, et al. Weightreducing effects of the plasma protein encoded by the obese gene. Science 1995; 269 : 543-6.

3. Schwartz MW, Woods SC, Porte D Jr, Seeley RJ, Baskin DG. Central nervous system control of food intake. Nature 2000; 404: 661-71.

4. Tartaglia LA, Dembski M, Weng $X$, et al. Identification and expression cloning of a leptin receptor, $\mathrm{Ob}-\mathrm{R}$. Cell 1995; 83: 1263-71.

5. Lee GH, Proenca R, Montez JM, et al. Abnormal splicing of the leptin receptor in diabetic mice. Nature 1996; 379: 632-5.

6. Masuzaki H, Ogawa $\mathrm{Y}$, Sagawa N, et al. Nonadipose tissue production of leptin: leptin as a novel placentaderived hormone in humans. Nat Med 1997; 3: 1029-33.

7. Wang J, Liu R, Hawkins M, Barzilai N, Rossetti L. A nutrient-sensing pathway regulates leptin gene expression in muscle and fat. Nature 1998; 393: 684-8.

8. Bado A, Levasseur $S$, Attoub $S$, et al. The stomach is a source of leptin. Nature 1998; 394: 790-3.
9. Sobhani I, Bado A, Vissuzaine $C$, et al. Leptin secretion and leptin receptor in the human stomach. Gut 2000; 47: 178-83.

10. Sobhani I, Buyse M, Goiot $\mathrm{H}$, et al. Stimulation rapidly increases leptin secretion in human stomach. Gastroenterology 2002; 122: 259-63.

11. Attoub S, Levasseur $S$, Buyse $M$, et al. Physiological role of cholecystokinin B/gastrin receptor in leptin secretion. Endocrinology 1999; 140: 4406-10.

12. Guilmeau $S$, Buyse $M$, Tsocas A, Laigneau JP, Bado A. Duodenal leptin stimulates cholecystokinin secretion: evidence of a positive leptincholecystokinin feedback loop. Diabetes 2003; 52: 1664-72.

13. Buyse M, Berlioz F, Guilmeau S, et al. PepTlmediated epithelial transport of dipeptides and cephalexin is enhanced by luminal leptin in the small intestine. J Clin Invest 2001; 108: 1483-94.

14. Barrenetxe J, Villaro AC, Guembe L, et al. Distribution of the long leptin receptor isoform in brush border, basolateral membrane, and cytoplasm of enterocytes. Gut 2002; 50 : 797-802.

15. Barrachina MD, Martinez V, Wang L, Wei Jy, Tache Y. Synergistic interaction between leptin and cholecystokinin to reduce short-term food intake in lean mice. Proc Natl Acad Sci USA 1997; 94: 10455-60.
16. Buyse M, Ovesjo ML, Goiot $H$, et al. Expression and regulation of leptin receptor proteins in afferent and efferent neurons of the vagus nerve. EurJ Neurosci 2001; 14: 64-72.

17. Burdyga G, Spiller D, Morris R, et al. Expression of the leptin receptor in rat and human nodose ganglion neurones. Neuroscience 2002; 109: 339-47.

18. Yuan CS, Attele AS, Dey L, Xie JT. Gastric effects of cholecystokinin and its interaction with leptin on brainstem neuronal activity in neonatal rats. J Pharmacol Exp Ther 2000; 295: 177-82.

19. Morton NM, Emilsson V, Liu YL, Cawthorne MA. Leptin action in intestinal cells. J Biol Chem 1998; 273: 26194-201.

20. Stan $S$, Levy $\varepsilon$, Bendayan $M$, et al. Effect of human recombinant leptin on lipid handling by fully differentiated Caco-2 cells. FEBS Lett 2001 ; 508: 80-4.

21. Lostao MP, Urdaneta $\varepsilon$, Martinez-Anso $\varepsilon$, Barber A, Martinez JA. Presence of leptin receptors in rat small intestine and leptin effect on sugar absorption. FEBS Lett 1998; 423: 302-6.

22. Fei $Y$ J, Kanai $y$, Nussberger $S$, et al. Expression cloning of a mammalian protoncoupled oligopeptide transporter. Nature 1994; 368: 563-6.
23. Siegmund B, Lehr $H A$, Fantuzzi G. Leptin: a pivotal mediator of intestinal inflammation in mice. Gastroenterology 2002; 122: 2011-25.

24. Sitaraman SV, Buyse $M$, Sun J, et al. Intestinal leptin: a novel proinflammatory cytokine. Gastroenterology 2002; 120: A879.

25. Buyse M, Sitaraman SV, Liu X, Bado A, Merlin D. Luminal leptin enhances CD147/MCT-1-mediated uptake of butyrate in the human intestinal cell line Caco2-BBE. J Biol Chem 2002; 277 : 28182-90.

26. Kirk P, Wilson MC, Heddle C, Brown MH, Barclay AN, Halestrap AP. CD147 is tightly associated with lactate transporters MCTl and MCT4 and facilitates their cell surface expression. EMBO J 2000; 19: 3896-904.

27. Mellinkoff $S$, Franklin $M$, Boyle D, Geipell M. Relationship between serum amino acid concentration and fluctuation in appetite. J Appl Physiol 1956; 8: 535.

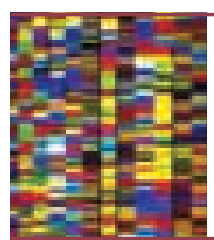

\section{Retrouvez chaque mois médecine/sciences sur France-Info dans la chronique «Info-Sciences 》 de Marie-Odile Monchicourt, du lundi au mercredi. france-info.com}

